\title{
HOMOGENEOUS RIGHT COIDEAL SUBALGEBRAS OF QUANTIZED ENVELOPING ALGEBRAS
}

\author{
I. HECKENBERGER AND S. KOLB
}

\begin{abstract}
For a quantized enveloping algebra of a complex semisimple Lie algebra with deformation parameter not a root of unity, we classify all homogeneous right coideal subalgebras. Any such right coideal subalgebra is determined uniquely by a triple consisting of two elements of the Weyl group and a subset of the set of simple roots satisfying some natural conditions. The essential ingredients of the proof are the Lusztig automorphisms and the classification of homogeneous right coideal subalgebras of the Borel Hopf subalgebras of quantized enveloping algebras obtained previously by H.-J. Schneider and the first named author.
\end{abstract}

\section{INTRODUCTION}

Unlike their classical analogs, quantized enveloping algebras do not admit many Hopf subalgebras. Folklore has it that Lie subalgebras of a, say, semisimple complex Lie algebra $\mathfrak{g}$ should possess quantum analogs which are coideal subalgebras of the corresponding quantized enveloping algebra $U_{q}(\mathfrak{g})$. Once this point of view was established in the early nineties, large classes of quantum group analogs of homogeneous spaces were constructed via (one-sided or two-sided) coideal subalgebras of $U_{q}(\mathfrak{g})$ [NS95, Dij96, Let02. All these constructions, however, are closely tied to the underlying classical situation.

In the present paper we follow a different route which aims at a general classification of right coideal subalgebras of $U_{q}(\mathfrak{g})$ under mild additional assumptions. More precisely, let $U^{0}$ denote the subalgebra generated by all group-like standard generators of $U_{q}(\mathfrak{g})$. We call a right coideal subalgebra of $U_{q}(\mathfrak{g})$ homogeneous if it contains $U^{0}$. In the present paper we determine all homogeneous right coideal subalgebras of $U_{q}(\mathfrak{g})$, for $q$ not a root of unity, in terms of pairs of elements of the corresponding Weyl group $W$. Our main result, Theorem 3.8 states the following:

There exists a one-to-one correspondence between the set of homogeneous right coideal subalgebras of $U_{q}(\mathfrak{g})$ and the set of triples $(x, u, J)$, where $x, u \in W$, the element $u^{-1}$ is a beginning of $x$, and $J$ is an arbitrary subset of $\Pi \cap x \Pi$, where $\Pi$ denotes a fixed basis of the root system of $\mathfrak{g}$.

Here we call $u^{-1}$ a beginning of $x$ if $u^{-1} \leq_{R} x$ with respect to the weak (or Duflo) order $\leq_{R}$ on $W$, see Section 2.3 for a precise definition.

Our theorem implies in particular that the number $|B(W)|$ of homogeneous right coideal subalgebras of $U_{q}(\mathfrak{g})$ depends only on $W$ and not explicitly on $\mathfrak{g}$. With

2000 Mathematics Subject Classification. 17B37; 81R50.

Key words and phrases. Quantum groups, coideal subalgebras, Weyl group, weak order.

The work of I. Heckenberger was supported by a Heisenberg professorship of the German Research Foundation (DFG). 
the help of the computer algebra program FELIX [AK91, AK] we determine the numbers $|B(W)|$ for all $W$ of order less than $10^{6}$. The largest such example is the Weyl group of type $B_{7}$ which has order 645120 . Our calculations confirm the results obtained previously by Kharchenko and Sagahon in KS08 for type $A_{n}$. However, our numbers differ from those given recently by Pogorelsky in type $G_{2}$ Pog11 and by Kharchenko, Sagahon, and Rivera in type $B_{n}$ with $n \geq 3$ [KSR11. We comment on these differences in Remark 3.12 .

The systematic investigation of homogeneous right coideal subalgebras of $U_{q}(\mathfrak{g})$ was initiated by Kharchenko in [KS08. This led to the astonishing conjecture that the number of homogeneous right coideal subalgebras of the Borel Hopf subalgebra $U^{\geq 0}$ of $U_{q}(\mathfrak{g})$ coincides with the order of the Weyl group $W$ of $\mathfrak{g}$. This conjecture provided the first indication that coideal subalgebras of $U_{q}(\mathfrak{g})$ can be classified in terms of Weyl group combinatorics. It was proved for $\mathfrak{g}$ of type $A_{n}$ and $B_{n}$ by Kharchenko [KS08, Kha11.

Recently, Kharchenko's conjecture was proved in a much wider context by H.J. Schneider and the first named author, namely for bosonizations $\mathcal{B}(V) \# H$ of Nichols algebras of semisimple Yetter-Drinfeld modules $V$ over an arbitrary Hopf algebra $H$ with bijective antipode [HS09. For $U_{q}(\mathfrak{g})$ they showed that the homogeneous right coideal subalgebras of $U^{\geq 0}$ are precisely the algebras $U^{+}[w] U^{0}$, where $U^{+}[w]$ denotes the quantum analog of a nilpotent Lie algebra introduced in CKP95] for any $w \in W$. The algebras $U^{+}[w]$ have recently also undergone a renaissance within the general program of investigation of prime spectra of quantum algebras, see for example Yak10. It is noteworthy that in the classification of prime ideals of $U^{+}[w]$ which are invariant under the adjoint action of $U^{0}$, the Bruhat (or strong) order plays an important role while the poset structure of homogeneous right coideal subalgebras of $U_{q}(\mathfrak{g})$ given by inclusion is identified with the weak order of $W$.

To describe homogeneous right coideal subalgebras of $U_{q}(\mathfrak{g})$ one uses the triangular decomposition of $U_{q}(\mathfrak{g})$. One observes that any homogeneous right coideal subalgebra $C$ of $U_{q}(\mathfrak{g})$ can be written as a product $C=C^{-} C^{+}$where $C^{-}$and $C^{+}$ are homogeneous right coideal subalgebras of the negative and the positive Borel Hopf subalgebras $U^{\leq 0}$ and $U^{\geq 0}$, respectively. As the homogeneous right coideal subalgebras of $U \leq 0$ and $U^{\geq 0}$ are known by the classification described above, it remains to determine when their product is a subalgebra. Again, Kharchenko et al. KS08, KSR11 were able to handle the series $A_{n}$ and $B_{n}$. However, their approach does not exhibit any obvious relation to the combinatorics of Weyl groups.

The classification of homogeneous right coideal subalgebras of $U_{q}(\mathfrak{g})$ presented in this paper is certainly not the end of the story. In HK11 we classified all right coideal subalgebras $C$ of $U^{\geq 0}$ for which $C \cap U^{0}$ is a Hopf algebra. In view of the results of the present paper it now seems feasible to classify right coideal subalgebras $C$ of $U_{q}(\mathfrak{g})$ under the weaker assumption that $C \cap U^{0}$ is a Hopf algebra. This class contains in particular all quantum symmetric pair coideal subalgebras defined in Let02. We expect that such a classification will conceptualize and simplify the understanding of many existing examples of right coideal subalgebras of $U_{q}(\mathfrak{g})$.

\section{Preliminaries}

2.1. Quantized enveloping algebras. We mostly follow the notation and conventions of Jan96. Let $\mathfrak{g}$ be a finite-dimensional complex semisimple Lie algebra 
and let $\Phi$ be the root system with respect to a fixed Cartan subalgebra. We also fix a basis $\Pi$ of $\Phi$ and denote by $\Phi^{+}$the corresponding set of positive roots. Let $W$ be the Weyl group of $\mathfrak{g}$ and let $(\cdot, \cdot)$ be the invariant scalar product on the real vector space generated by $\Pi$ such that $(\alpha, \alpha)=2$ for all short roots in each component. For any $\beta \in \Phi$ we write $s_{\beta}$ to denote the reflection at the hyperplane orthogonal to $\beta$ with respect to $(\cdot, \cdot)$. Let $Q=\mathbb{Z} \Pi$ be the root lattice. Let $U=U_{q}(\mathfrak{g})$ be the quantized enveloping algebra of $\mathfrak{g}$ in the sense of [Jan96. Chapter 4]. More precisely, let $k$ be a field and fix an element $q \in k$ with $q \neq 0$ and $q^{n} \neq 1$ for all $n \in \mathbb{N}$. Then $U$ is the unital associative algebra defined over $k$ with generators $K_{\alpha}, K_{\alpha}^{-1}, E_{\alpha}, F_{\alpha}$ for all $\alpha \in \Pi$ and relations given in [Jan96, 4.3]. By [Jan96, Proposition 4.11] there is a unique Hopf algebra structure on $U$ with coproduct $\Delta$, counit $\varepsilon$, and antipode $S$ such that

$$
\begin{aligned}
& \Delta\left(E_{\alpha}\right)=E_{\alpha} \otimes 1+K_{\alpha} \otimes E_{\alpha}, \quad \varepsilon\left(E_{\alpha}\right)=0, \quad S\left(E_{\alpha}\right)=-K_{\alpha}^{-1} E_{\alpha}, \\
& \Delta\left(F_{\alpha}\right)=F_{\alpha} \otimes K_{\alpha}^{-1}+1 \otimes F_{\alpha}, \quad \varepsilon\left(F_{\alpha}\right)=0, \quad S\left(F_{\alpha}\right)=-F_{\alpha} K_{\alpha}, \\
& \Delta\left(K_{\alpha}\right)=K_{\alpha} \otimes K_{\alpha}, \quad \varepsilon\left(K_{\alpha}\right)=1, \quad S\left(K_{\alpha}\right)=K_{\alpha}^{-1} .
\end{aligned}
$$

As in Jan96, Chapter 4] let $U^{+}, U^{-}$, and $U^{0}$ be the subalgebras of $U$ generated by the sets $\left\{E_{\alpha} \mid \alpha \in \Pi\right\},\left\{F_{\alpha} \mid \alpha \in \Pi\right\}$, and $\left\{K_{\alpha}, K_{\alpha}^{-1} \mid \alpha \in \Pi\right\}$, respectively. Moreover, let $U^{\geq 0}$ and $U \leq 0$ be the subalgebras of $U$ generated by the sets $\left\{K_{\alpha}, K_{\alpha}^{-1}, E_{\alpha} \mid \alpha \in\right.$ $\Pi\}$ and $\left\{K_{\alpha}, K_{\alpha}^{-1}, F_{\alpha} \mid \alpha \in \Pi\right\}$, respectively. In fact, $U^{0}$ and $U^{\geq 0}$ and $U^{\leq 0}$ are Hopf subalgebras of $U_{q}(\mathfrak{g})$. For any $\beta \in Q$ define $K_{\beta}=\prod_{\alpha \in \Pi} K_{\alpha}^{n_{\alpha}}$ if $\beta=\sum_{\alpha \in \Pi} n_{\alpha} \alpha$ for some $n_{\alpha} \in \mathbb{Z}$.

For any subspace $M$ of $U$ which is invariant under conjugation by all $K_{\alpha}$ for $\alpha \in \Pi$, we define the weight space $M_{\beta}$ of weight $\beta \in Q$ by

$$
M_{\beta}=\left\{m \in M \mid K_{\alpha} m K_{\alpha}^{-1}=q^{(\alpha, \beta)} m \text { for all } \alpha \in \Pi\right\} .
$$

We will apply this notation in particular if $M$ is one of $U, U^{+}$, or $U^{-}$. We call the elements of $M_{\beta}$ weight vectors of weight $\beta$ in $M$. For two subspaces $A, B$ of $U_{q}(\mathfrak{g})$ we write $A B$ for the $\operatorname{subspace}_{\operatorname{span}_{k}}\{a b \mid a \in A, b \in B\}$. For a subset $A \subseteq U_{q}(\mathfrak{g})$ we write $k\langle A\rangle$ for the unital subalgebra of $U_{q}(\mathfrak{g})$ generated by $A$.

2.2. The triangular decomposition. The algebra $U$ has a triangular decomposition in the sense that the multiplication map

$$
U^{-} \otimes U^{0} \otimes U^{+} \rightarrow U
$$

is an isomorphism of vector spaces. Recall from the introduction that we call a right coideal subalgebra of $U$ homogeneous if it contains $U^{0}$. The triangular decomposition of $U$ descends to the level of homogeneous right coideal subalgebras of $U$. This result is in principle contained in [Let02, Section 4] and was also proved in Kha10. Here we recall the argument for the convenience of the reader.

Proposition 2.1. Let $C$ be a homogeneous right coideal subalgebra of $U$. Then the multiplication map

$$
\left(U^{-} \cap C\right) \otimes U^{0} \otimes\left(U^{+} \cap C\right) \rightarrow C
$$

is an isomorphism of vector spaces.

Proof. In view of the triangular decomposition (2.4) of $U$ it suffices to show that the multiplication map (2.5) is surjective. To this end consider any weight vector 
$x \in C_{\beta}$ of weight $\beta \in Q$. Using the triangular decomposition (2.4) one can write

$$
x=\sum_{\mu, \alpha, i} F_{\beta-\alpha, \mu, i} K_{\mu} E_{\alpha, \mu, i}
$$

where for fixed $\mu, \alpha \in Q$, the elements of $\left\{E_{\alpha, \mu, i}\right\}$ are linearly independent weight vectors of weight $\alpha$ in $U^{+}$, and similarly, the elements of $\left\{F_{\beta-\alpha, \mu, i}\right\}$ are linearly independent weight vectors of weight $\beta-\alpha$ in $U^{-}$. The relations (2.1) - (2.3) imply that for any $\alpha, \mu, i$ one has

$$
\Delta\left(F_{\beta-\alpha, \mu, i} K_{\mu} E_{\alpha, \mu, i}\right) \in K_{\mu} E_{\alpha, \mu, i} \otimes F_{\beta-\alpha, \mu, i} K_{\mu}+\sum_{\gamma<\alpha} U_{\gamma} \otimes U_{\beta-\gamma} .
$$

Now choose $\alpha \in Q$ maximal such that $E_{\alpha, \mu, i} \neq 0 \neq F_{\beta-\alpha, \mu, i}$ for some $\mu, i$. By (2.6), the linear independence of the set $\left\{F_{\beta-\alpha, \mu, i} K_{\mu} \mid \mu \in Q\right\}$ and the maximality of $\alpha$ imply that $K_{\mu} E_{\alpha, \mu, i} \in C$ for all $\mu, i$. As $U^{0} \subseteq C$ one gets $E_{\alpha, \mu, i} \in C$. A similar argument proves that $F_{\beta-\alpha, \mu, i} \in C$ for all $\mu, i$. Now one obtains

$$
x \in\left(U^{-} \cap C\right) U^{0}\left(U^{+} \cap C\right)
$$

by induction on $\alpha$.

2.3. Homogeneous right coideal subalgebras of $U^{\geq 0}$. For any right coideal subalgebra $C$ of $U$ with $U^{0} \subseteq C$, Proposition 2.1 implies that $C=C^{-} C^{+}$where $C^{+}=\left(C \cap U^{+}\right) U^{0}$ and $C^{-}=\left(C \cap U^{-}\right) U^{0}$ are right coideal subalgebras of $U^{\geq 0}$ and $U \leq 0$, respectively, both containing $U^{0}$. Such coideal subalgebras of $U^{\geq 0}$ were classified in [HS09. In order to formulate this result, we first recall the Lusztig automorphisms $T_{\alpha}$ of $U$ defined for $\alpha \in \Pi$ in [Jan96, 8.14] and the quantum analogs $U^{+}[w]$ of nilpotent Lie algebras defined for any $w \in W$.

Let $w \in W$ be an element of length $\ell(w)=t$ and choose $\alpha_{1}, \ldots, \alpha_{t} \in \Pi$ such that $s_{\alpha_{1}} s_{\alpha_{2}} \cdots s_{\alpha_{t}}$ is a reduced expression of $w$. Until the end of this section we will keep these notations whenever we are in need of a reduced expression of an element $w \in W$. For all $i \in\{1,2, \ldots, t\}$ let $\beta_{i}=s_{\alpha_{1}} \cdots s_{\alpha_{i-1}} \alpha_{i}$. We define

$$
\Phi^{+}(w)=\left\{\alpha \in \Phi^{+} \mid w^{-1} \alpha<0\right\}
$$

and one verifies that $\Phi^{+}(w)=\left\{\beta_{i} \mid i=1, \ldots, t\right\}$. Moreover, one defines an automorphism $T_{w}=T_{\alpha_{1}} T_{\alpha_{2}} \cdots T_{\alpha_{t}}$ which by [Jan96, 8.18] is independent of the chosen reduced expression for $w$. The inverse of $T_{w}$ is obtained by

$$
T_{w}^{-1}=\tau \circ T_{w^{-1}} \circ \tau
$$

where $\tau$ is the involutive algebra antiautomorphism of $U$ determined by

$$
\tau\left(E_{\alpha}\right)=E_{\alpha}, \quad \tau\left(F_{\alpha}\right)=F_{\alpha}, \quad \tau\left(K_{\alpha}\right)=K_{\alpha}^{-1}, \quad \tau\left(K_{\alpha}^{-1}\right)=K_{\alpha},
$$

for all $\alpha \in \Pi$, see [Jan96, 8.18(6)]. The following Lemma will be useful to characterize right coideal subalgebras of $U \leq 0$ in the next subsection.

Lemma 2.2. Let $\mu=\sum_{\alpha \in \Pi} n_{\alpha} \alpha \in Q$ and $u \in U_{-\mu}^{-}$. Then $\tau(u)=c(\mu) S(u) K_{-\mu}$ where

$$
c(\mu)=(-1)^{\sum_{\alpha \in \Pi} n_{\alpha}} q^{\frac{1}{2}(\mu, \mu)-\frac{1}{2} \sum_{\alpha \in \Pi} n_{\alpha}(\alpha, \alpha)}
$$

only depends on $\mu$ and not on $u$. 
Proof. The lemma holds for $\mu=0$ and for $\mu \in \Pi$ by definition of $\tau$. Thus it holds for all $\mu$ since $\tau$ is an algebra antiautomorphism and

$$
\begin{aligned}
c\left(\mu_{1}\right) c\left(\mu_{2}\right) S\left(u_{2}\right) K_{-\mu_{2}} S\left(u_{1}\right) K_{-\mu_{1}} & =c\left(\mu_{1}\right) c\left(\mu_{2}\right) q^{\left(\mu_{1}, \mu_{2}\right)} S\left(u_{2}\right) S\left(u_{1}\right) K_{-\mu_{1}-\mu_{2}} \\
& =c\left(\mu_{1}+\mu_{2}\right) S\left(u_{1} u_{2}\right) K_{-\mu_{1}-\mu_{2}}
\end{aligned}
$$

for all $\mu_{1}, \mu_{2} \in Q$ and $u_{1} \in U_{-\mu_{1}}^{-}, u_{2} \in U_{-\mu_{2}}^{-}$.

In [Jan96, 8.21] Lusztig root vectors in $U^{+}$are defined by $E_{\beta_{i}}=T_{\alpha_{1}} \cdots T_{\alpha_{i-1}} E_{\alpha_{i}}$ for all $i \in\{1, \ldots, t\}$. It is known [Jan96, 8.21(1)] that $E_{\beta_{i}} \in U_{\beta_{i}}^{+}$for all $i \in$ $\{1, \ldots, t\}$. Moreover, if $\beta_{i} \in \Pi$ for some $i$ then $E_{\beta_{i}}$ coincides with the standard generator $E_{\beta_{i}}$ of $U^{+}$by [Jan96, 8.20]. Following [CKP95] the subspace

$$
U^{+}[w]=\operatorname{span}_{k}\left\{E_{\beta_{t}}^{a_{t}} \cdots E_{\beta_{2}}^{a_{2}} E_{\beta_{1}}^{a_{1}} \mid a_{1}, \ldots, a_{t} \in \mathbb{N}_{0}\right\}
$$

is attached to $w$ in [Jan96, 8.24]. It is shown in CKP95 that $U^{+}[w]$ is a subalgebra which does not depend on the reduced expression of $w$. For later purposes we recall the PBW-theorem for $U^{+}[w]$, see [Jan96, Chapter 8].

Proposition 2.3. The set

$$
\left\{E_{\beta_{1}}^{n_{1}} E_{\beta_{2}}^{n_{2}} \cdots E_{\beta_{t}}^{n_{t}} \mid n_{1}, n_{2}, \ldots, n_{t} \in \mathbb{N}_{0}\right\}
$$

is a basis of $U^{+}[w]$.

Definition (2.8) and Proposition 2.3) imply the following result.

Corollary 2.4. Let $x, y \in W$ with $\ell(x y)=\ell(x)+\ell(y)$. Then

$$
U^{+}[x y]=U^{+}[x] T_{x}\left(U^{+}[y]\right)=T_{x}\left(U^{+}[y]\right) U^{+}[x] .
$$

The algebras $U^{+}[w]$ for $w \in W$ are significant building blocks of right coideal subalgebras of $U \geq 0$. To make this statement precise, recall that the Weyl group is a poset with respect to the weak (or Duflo) order which we denote by $\leq_{R}$, see [BB06, Chapter 3]. By definition, one has $v \leq_{R} w$ for $v, w \in W$ if there exists $u \in W$ such that $w=v u$ and $\ell(w)=\ell(v)+\ell(u)$. On the other hand, the set of right coideal subalgebras of $U$ which contain $U^{0}$ is also a poset with respect to inclusion. The following theorem, which was proved in [HS09, Theorem 7.3], now explains the role of the algebras $U^{+}[w]$ in the investigation of homogeneous right coideal subalgebras of $U$.

Theorem 2.5. The map from $W$ to the set of right coideal subalgebras of $U \geq 0$ containing $U^{0}$, given by $w \mapsto U^{+}[w] U^{0}$, is an order preserving bijection.

As a first application of the above theorem we prove a Lemma which will be repeatedly used in Section 3

Lemma 2.6. Let $w \in W$ and $\alpha \in \Pi$.

(1) The following are equivalent.

(a) $E_{\alpha} \in U^{+}[w] U^{0}$

(b) $\ell\left(s_{\alpha} w\right)=\ell(w)-1$,

(c) $\alpha \in \Phi^{+}(w)$.

(2) Assume that $\ell\left(s_{\alpha} w\right)=\ell(w)+1$. The following are equivalent.

(a) The subspace $k\left\langle E_{\alpha}\right\rangle U^{+}[w] U^{0}$ of $U^{\geq 0}$ is a subalgebra,

(b) $\alpha \in \Pi \cap w \Pi$,

(c) There exists $\beta \in \Pi$ such that $s_{\alpha} w=w s_{\beta}$. 
Proof. (1) Property (b) implies (a) as $U^{+}[w]$ is independent of the chosen reduced expression for $w$. Moreover (b) implies (c) by definition of $\Phi^{+}(w)$. If $\ell\left(s_{\alpha} w\right)=$ $\ell(w)+1$, on the other hand, then $\Phi^{+}\left(s_{\alpha} w\right)=\{\alpha\} \cup s_{\alpha}\left(\Phi^{+}(w)\right)$. Since $\Phi^{+}\left(s_{\alpha} w\right) \subseteq$ $\Phi^{+}$, we conclude that $\alpha \notin \Phi^{+}(w)$. Consequently, $E_{\alpha} \notin U^{+}[w] U^{0}$ since $U^{+}[w] U^{0}$ is $\mathbb{Z} \Pi$-graded.

(2) Clearly, (b) is equivalent to (c) with $\beta=w^{-1} \alpha$, and (c) implies (a) by Corollary 2.4 since $w \beta=\alpha$. Assume now that (a) holds. By Theorem 2.5, since $k\left\langle E_{\alpha}\right\rangle U^{0}$ is a right coideal of $U^{\geq 0}$, there exists $v \in W$ such that $k\left\langle E_{\alpha}\right\rangle U^{+}[w] U^{0}=U^{+}[v] U^{0}$. Theorem [2.5 and $U^{+}[w] U^{0} \subseteq U^{+}[v] U^{0}$ imply that $w \leq_{R} v$. Proposition 2.3 yields now that $v=w s_{\beta}$ with $\beta \in \Pi, w \beta=\alpha$, and hence (b) holds.

2.4. Homogeneous right coideal subalgebras of $U \leq 0$. As a consequence of Theorem 2.5 we can immediately describe all homogeneous right coideal subalgebras of $U^{\leq 0}$. To this end consider the algebra automorphism $\omega$ of $U$ given by

$$
\omega\left(E_{\alpha}\right)=F_{\alpha}, \quad \omega\left(F_{\alpha}\right)=E_{\alpha}, \quad \omega\left(K_{\alpha}\right)=K_{\alpha}^{-1}
$$

for all $\alpha \in \Phi$. Following [Jan96, 8.24] one defines $U^{-}[w]=\omega\left(U^{+}[w]\right)$. Since $\omega$ is a coalgebra antiautomorphism, one concludes that $U^{-}[w] U^{0}=\omega\left(U^{+}[w] U^{0}\right)$ is a left coideal subalgebra of $U$. To obtain a right coideal subalgebra we consider $S\left(U^{-}[w] U^{0}\right)$. Theorem 2.5 implies the following result.

Corollary 2.7. Any right coideal subalgebra of $U^{\leq 0}$ which contains $U^{0}$ is of the form $S\left(U^{-}[w]\right) U^{0}$ for some $w \in W$.

In the remainder of this subsection we use the PBW-theorem for $U^{+}[w]$ and properties of the Lusztig automorphisms to obtain a suitable PBW-basis of $S\left(U^{-}[w]\right) U^{0}$. By [Jan96, Eq. 8.14(9)] one has

$$
T_{\alpha}(\omega(u)) \in k^{\times} \omega\left(T_{\alpha}(u)\right)
$$

for all $\alpha \in \Pi$ and all weight vectors $u \in U^{+}$, where $k^{\times}=k \backslash\{0\}$. In view of Proposition 2.3 this implies that $U^{-}[w]$ has a PBW-basis

$$
\left\{F_{\beta_{1}}^{n_{1}} F_{\beta_{2}}^{n_{2}} \ldots F_{\beta_{t}}^{n_{t}} \mid n_{1}, \ldots, n_{t} \in \mathbb{N}_{0}\right\}
$$

where $F_{\beta_{i}}=T_{\alpha_{1}} \cdots T_{\alpha_{i-1}} F_{\alpha_{i}}$ for all $i \in\{1,2, \ldots, t\}$. The following lemma will allow us to describe a PBW-basis of $S\left(U^{-}[w]\right) U^{0}$ without explicit use of the antipode.

Lemma 2.8. For any $w \in W$ one has

$$
T_{w}^{-1}\left(U^{+}[w] U^{0}\right)=S\left(U^{-}\left[w^{-1}\right]\right) U^{0} .
$$

Proof. We perform induction on the length $\ell(w)$ of $w$. If $\ell(w)=0$ then both sides of the above expression coincide with $U^{0}$. Now assume that there exists $\alpha \in \Pi$ such that $w=s_{\alpha} v$ with $\ell(w)=\ell(v)+1$. Then one calculates

$$
\begin{aligned}
T_{w}^{-1}\left(U^{+}[w] U^{0}\right) & =\left(T_{\alpha} T_{v}\right)^{-1}\left(k\left\langle E_{\alpha}\right\rangle T_{\alpha}\left(U^{+}[v]\right) U^{0}\right) \\
& =T_{v}^{-1}\left(k\left\langle F_{\alpha}\right\rangle U^{+}[v] U^{0}\right) .
\end{aligned}
$$

By induction hypothesis this implies the relation

$$
T_{w}^{-1}\left(U^{+}[w] U^{0}\right)=k\left\langle T_{v}^{-1} F_{\alpha}\right\rangle S\left(U^{-}\left[v^{-1}\right]\right) U^{0} .
$$


In view of the PBW-basis of $U^{-}\left[w^{-1}\right]$, see (2.10), it remains to show that $T_{v}^{-1} F_{\alpha} \in$ $S\left(T_{v^{-1}} F_{\alpha}\right) U^{0}$. Now, using (2.7), we obtain that

$$
\begin{aligned}
T_{v}^{-1} F_{\alpha} & =\tau \circ T_{v^{-1}} \circ \tau\left(F_{\alpha}\right) \\
& =\tau \circ T_{v^{-1}} F_{\alpha} \in S\left(T_{v^{-1}} F_{\alpha}\right) U^{0},
\end{aligned}
$$

where the last relation holds by Lemma 2.2. This proves the lemma.

Recall that we have fixed a reduced expression $w=s_{\alpha_{1}} s_{\alpha_{2}} \ldots s_{\alpha_{t}}$ of the element $w \in W$. We can now verify the desired PBW-theorem.

Corollary 2.9. The set

$$
\left\{\left(T_{\alpha_{1}}^{-1} \ldots T_{\alpha_{t-1}}^{-1} F_{\alpha_{t}}\right)^{n_{t}} \ldots\left(T_{\alpha_{1}}^{-1} F_{\alpha_{2}}\right)^{n_{2}} F_{\alpha_{1}}^{n_{1}} K_{\mu} \mid n_{1}, n_{2}, \ldots, n_{t} \in \mathbb{N}_{0}, \mu \in Q\right\}
$$

is a basis of $S\left(U^{-}[w]\right) U^{0}$.

Proof. Observe first that

$$
T_{w}^{-1}\left(T_{\alpha_{1}} T_{\alpha_{2}} \ldots T_{\alpha_{j-1}} E_{\alpha_{j}}\right)=-T_{\alpha_{t}}^{-1} T_{\alpha_{t-1}}^{-1} \ldots T_{\alpha_{j+1}}^{-1}\left(K_{\alpha_{j}}^{-1} F_{\alpha_{j}}\right) .
$$

Hence, in view of the PBW-basis of $U^{+}[w]$, Lemma 2.8 implies that the elements

$$
\left(T_{\alpha_{t}}^{-1} \ldots T_{\alpha_{2}}^{-1} F_{\alpha_{1}}\right)^{n_{t}} \ldots\left(T_{\alpha_{t}}^{-1} F_{\alpha_{t-1}}\right)^{n_{2}} F_{\alpha_{t}}^{n_{1}} K_{\mu}
$$

for $\left(n_{1}, \ldots, n_{t}\right) \in \mathbb{N}_{0}^{t}$ and $\mu \in Q$ form a basis of $S\left(U^{-}\left[w^{-1}\right]\right) U^{0}$. Now replace $w$ by $w^{-1}$ to obtain the claim of the corollary.

Lemma 2.8 moreover allows us to translate Corollary 2.4 into the setting of $U^{-}$.

Corollary 2.10. Let $x, y \in W$ with $\ell(x y)=\ell(x)+\ell(y)$. Then

$$
S\left(U^{-}[x y]\right) U^{0}=\left(T_{x^{-1}}^{-1} S\left(U^{-}[y]\right)\right) S\left(U^{-}[x]\right) U^{0}=S\left(U^{-}[x]\right)\left(T_{x^{-1}}^{-1} S\left(U^{-}[y]\right)\right) U^{0} .
$$

\section{Compatible pairs of Coideal subalgebras}

3.1. Classification. We summarize the main results of the previous section.

Proposition 3.1. $\quad$ (1) Any homogeneous right coideal subalgebra of $U$ is of the form $S\left(U^{-}[v]\right) U^{+}[w] U^{0}$ for some $v, w \in W$.

(2) The pair $(v, w)$ in $(1)$ is uniquely determined by the coideal subalgebra.

(3) For any $v, w \in W$ the subspace $S\left(U^{-}[v]\right) U^{+}[w] U^{0}$ is a right coideal subalgebra if and only if it is a subalgebra of $U$.

Proof. Property (1) follows from the triangular decomposition given by Proposition 2.1, from the classification in Theorem [2.5, and from Corollary 2.7 Property (2) holds because $U^{+}[w] \neq U^{+}\left[w^{\prime}\right]$ if $w \neq w^{\prime}$. Finally, Property (3) just expresses the fact that $S\left(U^{-}[v]\right) U^{+}[w] U^{0}$ is a right coideal which also holds by Theorem 2.5.

By the above proposition, to determine all homogeneous right coideal subalgebras of $U$ we need to determine the set

$$
A(W):=\left\{(v, w) \in W^{2} \mid S\left(U^{-}[v]\right) U^{+}[w] U^{0} \text { is a subalgebra of } U\right\} .
$$

To this end we first provide a few preparatory lemmas.

Lemma 3.2. Let $v, w \in W$. Then $(v, w) \in A(W)$ if and only if $(w, v) \in A(W)$. 
Proof. Observe that $S \circ \omega$ is an algebra antiautomorphism such that $(S \circ \omega)^{2}=$ id. Moreover, $S \circ \omega\left(U^{+}[w]\right)=S\left(U^{-}[w]\right)$ and $S \circ \omega\left(S\left(U^{-}[v]\right)\right)=\omega\left(U^{-}[v]\right)=U^{+}[v]$. Hence $S\left(U^{-}[v]\right) U^{+}[w] U^{0}$ is an algebra if and only if

$$
S \circ w\left(S\left(U^{-}[v]\right) U^{+}[w] U^{0}\right)=S\left(U^{-}[w]\right) U^{+}[v] U^{0}
$$

is an algebra.

Lemma 3.3. Let $(v, w) \in W^{2}$ and $\alpha \in \Pi$ such that $\ell\left(s_{\alpha} v\right)=\ell(v)+1$ and $\ell\left(s_{\alpha} w\right)=$ $\ell(w)+1$. Then $\left(s_{\alpha} v, w\right) \in A(W)$ if and only if $\left(v, s_{\alpha} w\right) \in A(W)$.

Proof. As $T_{\alpha}$ is an algebra automorphism of $U$, one has $\left(s_{\alpha} v, w\right) \in A(W)$ if and only if $T_{\alpha}\left(S\left(U^{-}\left[s_{\alpha} v\right]\right) U^{+}[w] U^{0}\right)$ is a subalgebra of $U$. Using Proposition 2.3 and Corollary 2.10 one obtains that

$$
T_{\alpha}\left(S\left(U^{-}\left[s_{\alpha} v\right]\right) U^{+}[w] U^{0}\right)=S\left(U^{-}[v]\right) U^{+}\left[s_{\alpha} w\right] U^{0}
$$

which completes the proof of the lemma.

Lemma 3.4. Let $(v, w) \in W^{2}$ and $\alpha \in \Pi$ such that $\ell\left(s_{\alpha} v\right)=\ell(v)+1$ and $\ell\left(s_{\alpha} w\right)=$ $\ell(w)+1$. Assume that $\left(s_{\alpha} v, s_{\alpha} w\right) \in A(W)$. Then $\alpha \in v \Pi \cap w \Pi$.

Proof. The assumption $\ell\left(s_{\alpha} v\right)=\ell(v)+1$ implies that $F_{\alpha} \in S\left(U^{-}\left[s_{\alpha} v\right]\right) U^{0}$. Hence $\left(s_{\alpha} v, s_{\alpha} w\right) \in A(W)$ implies that $k\left\langle F_{\alpha}\right\rangle U^{+}\left[s_{\alpha} w\right] U^{0}$ is a subalgebra of $U$. Hence

$$
T_{\alpha}^{-1}\left(k\left\langle F_{\alpha}\right\rangle U^{+}\left[s_{\alpha} w\right] U^{0}\right)=k\left\langle E_{\alpha}, F_{\alpha}\right\rangle U^{+}[w] U^{0}
$$

is a subalgebra of $U$. This is only possible if $k\left\langle E_{\alpha}\right\rangle U^{+}[w] U^{0}$ is a subalgebra of $U \geq 0$. Hence $\alpha \in w \Pi$ by Lemma 2.6. The statement about $v$ follows from the above argument and Lemma 3.2 .

For any subset $J \subseteq \Pi$ we write $w_{J}$ to denote the longest element of the parabolic subgroup $W_{J}$ corresponding to $J$. For any $v \in W$ define

$$
J_{v}=\left\{\alpha \in \Pi \mid \ell\left(s_{\alpha} v\right)<\ell(v)\right\} .
$$

Lemma 3.5. Let $v \in W$ and set $J=J_{v}$. Assume that for any $\alpha \in J$ there exists $\beta \in \Pi$ such that $v s_{\beta}=s_{\alpha} v$. Then $v=w_{J}$.

Proof. Lemma 2.6(2) with $w=s_{\alpha} v$ implies that $v^{-1} \alpha \in-\Pi$ for all $\alpha \in J$. Let $K=\left\{-v^{-1} \alpha \mid \alpha \in J\right\}$ and $u=w_{J} v$. Then $K \subseteq \Pi$ and $u^{-1} \alpha \in \Pi$ for all $\alpha \in J$. Let $\beta \in \Pi \backslash J$ and let $\gamma \in \sum_{\alpha \in J} \mathbb{N}_{0} \alpha$ such that $w_{J}^{-1} \beta=\beta+\gamma$. Then

$$
u^{-1} \beta=v^{-1}(\beta+\gamma) \in v^{-1} \beta-\sum_{\alpha \in K} \mathbb{N}_{0} \alpha .
$$

Since $\beta \notin J=J_{v}$, we conclude that $v^{-1} \beta \in \Phi^{+}$. Further, since $\beta \notin \sum_{\alpha \in J} \mathbb{Z} \alpha$, also $v^{-1} \beta \notin \sum_{\alpha \in K} \mathbb{N}_{0} \alpha$ holds. Thus $u^{-1} \beta \in \Phi^{+}$. Therefore $u^{-1} \Pi \subseteq \Phi^{+}$, that is, $\ell(u)=0$. Since $w_{J}=w_{J}^{-1}$, this implies that $v=w_{J}$.

Proposition 3.6. Let $(v, w) \in A(W)$. Then there exist elements $u, x \in W$ and $a$ subset $J \subseteq \Pi$ such that the following properties hold:

(1) $v=u w_{J}$ and $w=u w_{J} x$.

(2) $J \subseteq \Pi \cap x \Pi$.

(3) $u^{-1} \leq_{R} x$. 
Moreover, the triple $(x, u, J)$ is uniquely determined by the pair $(v, w)$ and one has

$$
\begin{aligned}
& \ell(v)=\ell(u)+\ell\left(w_{J}\right), \\
& \ell(w)=\ell(x)+\ell\left(w_{J}\right)-\ell(u) .
\end{aligned}
$$

Proof. Choose $u \leq_{R} v$ maximal such that $\ell\left(u^{-1} w\right)=\ell(u)+\ell(w)$. By Lemma 3.3 one has $\left(u^{-1} v, u^{-1} w\right) \in A(W)$. Define $J=J_{u^{-1} v}$. By choice of $u$ one has

$$
\ell\left(s_{\alpha} u^{-1} w\right)=\ell\left(u^{-1} w\right)-1 \quad \text { for any } \alpha \in J .
$$

This and Lemma3.4yield that $\alpha \in s_{\alpha} u^{-1} v \Pi$ for all $\alpha \in J$. By Lemma2.6.2), for all $\alpha \in J$ there exists $\beta \in \Pi$ such that $u^{-1} v=s_{\alpha} u^{-1} v s_{\beta}$, that is, $s_{\alpha} u^{-1} v=u^{-1} v s_{\beta}$. Then Lemma 3.5 implies that $u^{-1} v=w_{J}$. In particular, Equation (3.1) holds. Moreover, Equation (3.3) implies that $w_{J} \leq_{R} u^{-1} w$. Hence there exists $x \in W$ such that $u^{-1} w=w_{J} x$ and $\ell\left(w_{J}\right)+\ell(x)=\ell\left(u^{-1} w\right)$. In particular, property (1) holds and Equation (3.2) is satisfied by the choice of $u$.

To verify (2), choose $\alpha \in J$ and write $w_{J} x=s_{\alpha} y$ with $\ell\left(w_{J} x\right)=\ell(y)+1$. As $\left(w_{J}, w_{J} x\right) \in A(W)$, Lemma 3.4 implies that $\alpha \in y \Pi$. Hence $-\alpha \in w_{J} x \Pi$, and hence $-w_{J} \alpha \in x \Pi$. Since $-w_{J} J=J$, property (2) holds.

To prove (3), we first conclude from (2) and from Lemma 2.6(2) that there exists $K \subseteq \Pi$ with $x K=J$ and $w_{J} x=x w_{K}$. By the choice of $u$ and $J$ we obtain that $\ell\left(u s_{\alpha}\right)=\ell(u)+1$ for all $\alpha \in J$ and hence $u J \subseteq \Phi^{+}$. Thus $u x K=u J \subseteq \Phi^{+}$, and therefore

$$
\ell(w)=\ell\left(u w_{J} x\right)=\ell\left(u x w_{K}\right)=\ell(u x)+\ell\left(w_{K}\right)=\ell(u x)+\ell\left(w_{J}\right) .
$$

Hence $\ell(u x)=\ell(x)-\ell(u)$ by Equation (3.2), that is, property (3) holds.

To prove uniqueness of the triple $(x, u, J)$, observe first that $x=v^{-1} w$ is uniquely determined by $v$ and $w$. Define $M=\Pi \cap x \Pi$. Then $J \subseteq M$ by (2). Moreover, $\ell\left(x^{-1} s_{\alpha}\right)=\ell\left(x^{-1}\right)+1$ for all $\alpha \in M$. Hence, $x^{-1}$ is a minimal length left coset representative of $x^{-1} W_{M} \in W / W_{M}$. Property (3) implies that $u$ is a minimal length left coset representative of $u W_{M} \in W / W_{M}$. Therefore $u$ and $w_{J}$ are uniquely determined by $v=u w_{J}$, see [BB06, Proposition 2.4.4].

Let $P(\Pi)$ denote the power set of $\Pi$. Motivated by the above proposition we define a subset $B(W) \subseteq W^{2} \times P(\Pi)$ by

$$
B(W)=\left\{(x, u, J) \in W^{2} \times P(\Pi) \mid J \subseteq \Pi \cap x \Pi \text { and } u^{-1} \leq_{R} x\right\} .
$$

We now show that we have a well defined map from $B(W)$ to $A(W)$.

Proposition 3.7. If $(x, u, J) \in B(W)$ then $\left(u w_{J}, u w_{J} x\right) \in A(W)$.

Proof. Let $(x, u, J) \in B(W)$. As $u^{-1} \leq_{R} x$ and $\ell\left(s_{\alpha} x\right)=\ell(x)+1$ for all $\alpha \in J$ one has $\ell\left(w_{J} x\right)=\ell\left(w_{J}\right)+\ell(x)$ and $\ell\left(u w_{J}\right)=\ell(u)+\ell\left(w_{J}\right)$. Moreover, $J \subseteq \Pi \cap x \Pi$ and hence for all $\alpha \in J$ there exists $\beta \in \Pi$ with $s_{\alpha} x=x s_{\beta}$ by Lemma 2.6(2). This implies that $w_{J} x=x w_{K}$ for some subset $K \subseteq \Pi$. Hence $u w_{J} x=u x w_{K}$. Together with $\ell\left(w_{J} x\right)=\ell\left(w_{J}\right)+\ell(x)=\ell(x)+\ell\left(w_{K}\right)$ this implies that

$$
\ell\left(u x w_{K}\right)=\ell(x)-\ell(u)+\ell\left(w_{J}\right)=\ell\left(w_{J} x\right)-\ell(u) .
$$

Hence we can apply Lemma 3.3 and it suffices to show that $\left(w_{J}, w_{J} x\right) \in A(W)$.

Now, since $w_{J} x=x w_{K}$ and $x K=J$, we conclude that $T_{x}\left(U^{+}\left[w_{K}\right]\right)=U^{+}\left[w_{J}\right]$ and hence Corollary 2.4 implies that

$$
U^{+}\left[w_{J} x\right]=U^{+}[x] U^{+}\left[w_{J}\right] .
$$


To prove the proposition note that $U^{+}\left[w_{J}\right] T_{w_{J}}\left(U^{+}[x]\right)=U^{+}\left[w_{J} x\right]$ is an algebra. Hence Lemma 2.8 implies that $T_{w_{J}}\left(S\left(U^{-}\left[w_{J}\right]\right) U^{+}[x] U^{0}\right)$ and hence also $S\left(U^{-}\left[w_{J}\right]\right) U^{+}[x] U^{0}$ are algebras. Therefore $S\left(U^{-}\left[w_{J}\right]\right) U^{+}[x] U^{+}\left[w_{J}\right] U^{0}$ is an algebra. By (3.4) this proves that $\left(w_{J}, w_{J} x\right) \in A(W)$.

The following theorem summarizes Propositions 3.6 and 3.7. It states that the set $B(W)$ parametrizes the set of homogeneous right coideal subalgebras of $U$.

Theorem 3.8. The map $B(W) \rightarrow A(W)$ given by $(x, u, J) \mapsto\left(u w_{J}, u w_{J} x\right)$ is a bijection.

For any subset $J \subseteq \Pi$ let $U_{q}\left(\mathfrak{k}_{J}\right)$ denote the Hopf subalgebra of $U_{q}(\mathfrak{g})$ generated by $U^{0}$ and by $E_{\alpha}, F_{\alpha}$ with $\alpha \in J$. By Propositions 3.1 and 3.7 with $u=e$, for any $x \in W$ and any $J \subseteq \Pi \cap x \Pi$ the subspace $S\left(U^{-}\left[w_{J}\right]\right) U^{+}\left[w_{J} x\right] U^{0}$ is a homogeneous right coideal subalgebra of $U_{q}(\mathfrak{g})$. By Equation (3.4) this is equivalent to $U_{q}\left(\mathfrak{k}_{J}\right) U^{+}[x]$ being a homogeneous right coideal subalgebra of $U_{q}(\mathfrak{g})$.

Corollary 3.9. For any homogeneous right coideal subalgebra $C$ of $U_{q}(\mathfrak{g})$ there exist $x \in W$ and $J \subseteq \Pi \cap x \Pi$ such that $C$ is isomorphic as an algebra to $U_{q}\left(\mathfrak{k}_{J}\right) U^{+}[x]$.

Proof. By Theorem 3.8 any homogeneous right coideal subalgebra of $U_{q}(\mathfrak{g})$ is of the form $S\left(U^{-}\left[u w_{J}\right]\right) U^{+}\left[u w_{J} x\right] U^{0}$ for some $x, u \in W$ and $J \subseteq \Pi \cap x \Pi$, where $u^{-1} \leq_{R} x$. Since $u \leq_{R} u w_{J}$, we conclude from Corollary 2.10 that

$$
S\left(U^{-}\left[u w_{J}\right]\right) U^{0}=T_{u^{-1}}^{-1}\left(S\left(U^{-}\left[w_{J}\right]\right)\right) S\left(U^{-}[u]\right) U^{0} .
$$

Therefore

$$
\begin{aligned}
T_{u^{-1}} & \left(S\left(U^{-}\left[u w_{J}\right]\right) U^{+}\left[u w_{J} x\right] U^{0}\right) \\
& =T_{u^{-1}}\left(T_{u^{-1}}^{-1}\left(S\left(U^{-}\left[w_{J}\right]\right)\right) S\left(U^{-}[u]\right) U^{0} U^{+}\left[u w_{J} x\right]\right) \\
& =S\left(U^{-}\left[w_{J}\right]\right) U^{+}\left[u^{-1}\right] U^{0} T_{u^{-1}}\left(U^{+}\left[u w_{J} x\right]\right) \\
& =S\left(U^{-}\left[w_{J}\right]\right) U^{+}\left[w_{J} x\right] U^{0}
\end{aligned}
$$

where the second equation holds by Lemma 2.8 and the third by Corollary 2.4 since $\ell\left(u w_{J} x\right)=\ell\left(w_{J} x\right)-\ell(u)$ by Equation (3.2). The last expression equals $U_{q}\left(\mathfrak{k}_{J}\right) U^{+}[x]$ by Equation (3.4).

3.2. Calculations. By Theorem 3.8 the number of homogeneous right coideal subalgebras of $U$ coincides with the cardinality $|B(W)|$ of the set $B(W)$. The calculation of $|B(W)|$ is straightforward. For any $x \in W$ one has to determine the cardinality of the set $\left\{u \in W \mid u^{-1} \leq_{R} x\right\}$ and multiply by $2^{|\Pi \cap x \Pi|}$ to account for the choice of $J$. Finally one has to sum over all $x \in W$. In formulas, this gives

$$
|B(W)|=\sum_{x \in W}\left|\left\{u \in W \mid u \leq_{R} x\right\}\right| \cdot 2^{|\Pi \cap x \Pi|} .
$$

As $\Pi \cap x \Pi=\left\{\beta \in \Pi \mid \exists \alpha \in \Pi\right.$ such that $\left.s_{\beta}=x s_{\alpha} x^{-1}, \ell\left(x s_{\alpha}\right)=\ell(x)+1\right\}$, the cardinality $|B(W)|$ is given purely in terms of the Weyl group. This proves the following result.

Corollary 3.10. The number of homogeneous right coideal subalgebras of $U$ depends only on the Weyl group $W$ of $\mathfrak{g}$ as a Coxeter group but not explicitly on $\mathfrak{g}$. 


\begin{tabular}{|c|c|c|}
\hline$x$ & $\left|\left\{u \in W \mid u \leq_{R} x\right\}\right|$ & $|\Pi \cap x \Pi|$ \\
\hline$e$ & 1 & 2 \\
$s_{1}$ & 2 & 0 \\
$s_{2}$ & 2 & 0 \\
$s_{1} s_{2}$ & 3 & 0 \\
$s_{2} s_{1}$ & 3 & 0 \\
$s_{1} s_{2} s_{1}$ & 4 & 0 \\
$s_{2} s_{1} s_{2}$ & 4 & 0 \\
$s_{1} s_{2} s_{1} s_{2}$ & 5 & 0 \\
$s_{2} s_{1} s_{2} s_{1}$ & 5 & 1 \\
$s_{1} s_{2} s_{1} s_{2} s_{1}$ & 6 & 1 \\
$s_{2} s_{1} s_{2} s_{1} s_{2}$ & 6 & 0 \\
$s_{1} s_{2} s_{1} s_{2} s_{1} s_{2}$ & 12 & \\
\hline
\end{tabular}

Example 3.11. Let $\mathfrak{g}$ be of type $G_{2}$. For simplicity we denote the standard generators of $W$ by $s_{1}$ and $s_{2}$. In Table 1 we have recorded the numbers $\left|\left\{u \in W \mid u \leq_{R} x\right\}\right|$ and $|\Pi \cap x \Pi|$ for all $x \in W$. A straightforward calculation using (3.5) then yields $|B(W)|=68$. This calculation corrects the number given in [Pog11, see also Remark 3.12

It is possible to perform calculations as in Example 3.11 by hand for $W$ of types $A_{2}, A_{3}, B_{2}$, and $B_{3}$. To obtain the number $|B(W)|$ also for examples with larger Weyl groups we have used the computer algebra program FELIX AK91, AK. This allowed us to determine $|B(W)|$ for all Weyl groups of cardinality less than $10^{6}$. The results are displayed in Table 2, Our calculations confirm the results obtained in $\mathrm{KS} 08$ for type $A_{n}$.

Remark 3.12. In Pog11, Pogorelsky applies Kharchenko's method to determine the homogeneous right coideal subalgebras of $U_{q}(\mathfrak{g})$ and of the related small quantum group for $\mathfrak{g}$ of type $G_{2}$. She obtains a number different from ours due to a hidden mistake in her previous work $\mathrm{Pog} 09$ on right coideal subalgebras of the standard Borel Hopf subalgebra of $U_{q}(\mathfrak{g})$.

In [KSR11, Kharchenko, Sagahon and Rivera calculate the number of homogeneous right coideal subalgebras of $U_{q}(\mathfrak{g})$ for $\mathfrak{g}$ of type $B_{n}$ with $n \geq 2$. Again, the numbers for $n \geq 3$ are different from (more precisely, smaller than) the numbers given in Table 2, We have checked the cases $B_{3}$ and $B_{4}$ and confirm that both our description and the theory in KSR11 provide the same set of right coideal subalgebras and consequently the same numbers, that is, 664 for $B_{3}$ and 17848 for $B_{4}$. Hence there seems to be an error in the explicit computer calculations in KSR11.

\section{REFERENCES}

[AK] J. Apel and U. Klaus, FELIX, (\protect \vrule width0pt \protect \href \{http://felix.hgb-leipzig.de\}\{http://felix.

[AK91] , FELIX - an assistant for algebraists, ISSAC'91 (New York) (S.M. Watt, ed.), ACM Press, 1991, pp. 382-389.

[BB06] A. Björner and F. Brenti, Combinatorics of Coxeter groups, Springer-Verlag, New York, 2006. 


\begin{tabular}{|r|r|r|}
\hline$W$ & $|W|$ & $|B(W)|$ \\
\hline$A_{1}$ & 2 & 4 \\
$A_{2}$ & 6 & 26 \\
$A_{3}$ & 24 & 252 \\
$A_{4}$ & 120 & 3368 \\
$A_{5}$ & 720 & 58810 \\
$A_{6}$ & 5040 & 1290930 \\
$A_{7}$ & 40320 & 34604844 \\
$A_{8}$ & 362880 & 1107490596 \\
\hline$B_{2}$ & 8 & 38 \\
$B_{3}$ & 48 & 664 \\
$B_{4}$ & 384 & 17848 \\
$B_{5}$ & 3840 & 672004 \\
$B_{6}$ & 46080 & 33369560 \\
$B_{7}$ & 645120 & 2094849020 \\
\hline$D_{4}$ & 192 & 6512 \\
$D_{5}$ & 1920 & 238720 \\
$D_{6}$ & 23040 & 11633624 \\
$D_{7}$ & 322560 & 720453984 \\
\hline$E_{6}$ & 51840 & 38305190 \\
\hline$F_{4}$ & 1152 & 91244 \\
\hline$G_{2}$ & 12 & 68 \\
\hline
\end{tabular}

TABLE 2. Number of homogeneous right coideal subalgebras of $U$

[CKP95] C. De Concini, V.G. Kac, and C. Procesi, Some quantum analogues of solvable Lie groups, Geometry and analysis (Bombay 1992) (Bombay), Tata Inst. Fund. Res., 1995, pp. 41-65.

[Dij96] M.S. Dijkhuizen, Some remarks on the construction of quantum symmetric spaces, Acta Appl. Math. 44 (1996), no. 1-2, 59-80.

[HK11] I. Heckenberger and S. Kolb, Right coideal subalgebras of the Borel part of a quantized enveloping algebra, Int. Math. Res. Not. IMRN 2011 (2011), no. 2, 419-451.

[HS09] I. Heckenberger and H.-J. Schneider, Right coideal subalgebras of Nichols algebras and the Duflo order on the Weyl groupoid, Preprint, arXiv:0909.0293 (2009), 43 pp.

[Jan96] J.C. Jantzen, Lectures on quantum groups, Grad. Stud. Math., vol. 6, Amer. Math. Soc, Providence, RI, 1996.

[Kha10] V. K. Kharchenko, Triangular decomposition of right coideal subalgebras, J. Algebra 324 (2010), no. 11, 3048-3089.

[Kha11] , Right coideal subalgebras in $U_{q}^{+}\left(\mathfrak{s o}_{2 n+1}\right)$, J. Eur. Math. Soc. (JEMS) 13 (2011), 1675-1733.

[KS08] V. K. Kharchenko and A. V. Lara Sagahon, Right coideal subalgebras in $U_{q}\left(\mathfrak{s l}_{n+1}\right)$, J. Algebra 319 (2008), no. 6, 2571-2625.

[KSR11] V. K. Kharchenko, A. V. Lara Sagahon, and J. L. Garza Rivera, Computing of the number of right coideal subalgebras of $U_{q}\left(\mathfrak{s o}_{2 n+1}\right)$, J. Algebra 341 (2011), no. 1, 279296.

[Let02] G. Letzter, Coideal subalgebras and quantum symmetric pairs, New directions in Hopf algebras (Cambridge), MSRI publications, vol. 43, Cambridge Univ. Press, 2002, pp. 117166.

[NS95] M. Noumi and T. Sugitani, Quantum symmetric spaces and related q-orthogonal polynomials, Group theoretical methods in physics (Singapore) (A. Arima et. al., ed.), World Scientific, 1995, pp. 28-40. 
[Pog09] B. Pogorelsky, Right coideal subalgebras of the quantum Borel algebra of type $G_{2}$, J. Algebra 322 (2009), no. 7, 2335-2354.

[Pog11] - Right coideal subalgebras of quantized universal enveloping algebras of type $G_{2}$, Comm. Algebra 139 (2011), no. 4, 1181-1207.

[Yak10] M. Yakimov, Invariant prime ideals in quantizations of nilpotent Lie algebras, Proc. London Math. Soc. 101 (2010), no. 2, 454-476.

Istvan Heckenberger, Fachbereich Mathematik und Informatik, Philipps-Universität Marburg, Hans-Meerwein-Strasse, D-35032 Marburg, Germany

E-mail address: heckenberger@mathematik.uni-marburg.de

Stefan Kolb, School of Mathematics and Statistics, Newcastle University, NewcasTLE UPON TYNE NE1 7RU, UK

E-mail address: stefan.kolb@ncl.ac.uk 